\title{
Healthcare Encounters Pre-specified
}

National Cancer Institute

\section{Source}

National Cancer Institute. Healthcare Encounters Pre-specified. NCI Thesaurus. Code C123989.

An indication or description that a healthcare encounter was previously determined, characterized, or detailed. 